\title{
The impact of blood culture reporting and clinical liaison on the empiric treatment of bacteraemia
}

\author{
R J Cunney, E B McNamara, N Alansari, B Loo, E G Smyth
}

\begin{abstract}
Aims-To assess the impact of blood culture results and early clinical liaison on the treatment of patients with bacteraemia.

Methods-123 patients with significant positive blood cultures were followed over a nine month period in a 620 bed teaching hospital. The impact of early blood culture reporting and clinical liaison on the cost and appropriateness of treatment was assessed.
\end{abstract}

Results-Empiric treatment was started before the Gram stain result in 107 (87\%) patients. Treatment was altered on the basis of the Gram stain result in 39 (36\%) of these patients, and on culture and sensitivity results in $53(50 \%)$. The spectrum of antibiotic treatment was narrowed in $58(54 \%)$ of these; $20(19 \%)$ on Gram stain result alone. This resulted in a $42 \%$ reduction in daily antibiotic costs in patients who had received empiric treatment. Empiric treatment did not follow the hospital antibiotic policy in $49(46 \%)$ of the patients treated. In patients where empiric treatment was not in accordance with hospital policy, 21 (44\%) had an isolate resistant to the empiric treatment used; while in patients who received agents in accordance with hospital policy only one $(1.7 \%)$ had a resistant isolate $(p<0.05)$. Patients who died $(11(9 \%))$ were less likely to have received empiric treatment in accordance with the antibiotic policy, although this did not reach statistical significance $(p=0.1)$.

Conclusion-Early reporting of Gram stain results from blood cultures, combined with early clinical liaison, results in more rational and cost effective treatment.

(F Clin Pathol 1997;50:1010-1012)

Keywords: blood culture; bacteraemia; clinical liaison; cost effectiveness; audit

Audit of routine laboratory and clinical practice has become increasingly important. Critical assessment of these practices may reveal areas of deficiency and thus lead to improvement in the delivery of service. Validation of the clinical impact of such practices gives a useful educational and bargaining tool when dealing with fellow clinicians and management.

In this institution all patients with clinically significant blood culture isolates are routinely reviewed by members of the clinical microbiol- ogy team. After clinical assessment, further investigations and management are advised in consultation with the primary care team responsible for the patient. This form of laboratory-ward liaison is a well established practice but has rarely been audited. The clinical impact of laboratory data has been questioned, particularly when broad spectrum empirical antimicrobial treatment is available. ${ }^{1}$ We undertook a prospective analysis of our blood culture reporting protocol with particular reference to our hospital antibiotic policy. This policy gives guidance on empiric antibiotic treatment in light of the probable source of infection. The policy is produced under the guidance of the consultant clinical microbiologist, with contributions on individual categories of infection from relevant clinicians. It is approved by each clinical consultant before distribution to all non-consultant hospital doctors. Educational follow up is given at induction days for new interns and at medical and surgical grand rounds. This study was undertaken just before the biannual update of the policy.

\section{Methods}

Data was gathered prospectively on patients with clinically significant isolates recovered from blood over a nine month period. Blood cultures were processed using the Bactec NR-730 system (Becton-Dickinson Microbiology Systems, Sparks, Maryland, USA) and identified by standard microbiological techniques. Patients were reviewed by members of the clinical microbiology team. In addition to demographic and microbiological data, the source of bacteraemia was noted together with any significant underlying illness. Choice of agent(s), dose, frequency, and route of administration of empiric treatment was noted. The appropriateness of this treatment was assessed in relation to the organism(s) isolated, the underlying source, and the recommendations of the hospital antibiotic policy. Details were also recorded of changes in treatment recommended on the basis of Gram stain result and further changes made on the basis of culture and sensitivity results. Data were analysed using the Student's $t$ test.

\section{Results}

A total of 123 patients with significant positive blood cultures were analysed over a nine month period. There were 132 organisms isolated; nine blood cultures $(7 \%)$ had more than one isolate (table 1 ). The average time to detection of growth in blood culture bottles, at
Accepted for publication 10 October 1997 
Table 1 Organisms isolated and source $(n=132)$

\begin{tabular}{|c|c|c|c|c|c|c|c|}
\hline \multirow[b]{2}{*}{ Organism } & \multirow[b]{2}{*}{ No. (\%) } & \multicolumn{6}{|c|}{ Source } \\
\hline & & $U T$ & $C P$ & $A B$ & $W S$ & $R T$ & $O T$ \\
\hline Escherichia coli & $36(27)$ & 24 & 1 & 4 & 2 & - & 5 \\
\hline Staphylococcus aureus & $31(23)$ & - & 12 & 1 & 7 & 1 & 10 \\
\hline Streptococcus pneumoniae & $15(11)$ & - & - & - & - & 12 & 3 \\
\hline Coagulase negative staphylococci & $9(7)$ & - & 3 & 3 & - & 2 & 1 \\
\hline Enterococcus spp & $6(5)$ & 3 & 1 & 2 & - & - & - \\
\hline Pseudomonas spp & $6(5)$ & 4 & - & - & - & 1 & 1 \\
\hline Proteus spp & $5(4)$ & 4 & - & 1 & - & - & - \\
\hline Enterobacter $\mathrm{spp}$ & $4(3)$ & 1 & 2 & - & - & - & 1 \\
\hline Acinetobacter spp & $3(2)$ & 1 & 2 & - & - & - & - \\
\hline Haemophilus spp & $2(2)$ & - & - & - & - & 2 & - \\
\hline Bacillus sp & $1(1)$ & - & 1 & - & - & - & - \\
\hline Candida albicans & $1(1)$ & - & - & - & - & - & 1 \\
\hline Other streptococci & $7(5)$ & 2 & 2 & 1 & 1 & - & 1 \\
\hline Other enterobacteraeciae & $6(5)$ & 2 & - & 1 & - & - & 3 \\
\hline
\end{tabular}

$\mathrm{UT}$, urinary tract; $\mathrm{CP}$, central or peripheral venous cannula; $\mathrm{AB}$, abdominal or hepatobiliary; WS, wound or soft tissue; RT, respiratory tract; OT, other or unknown source.

which point a Gram stain was performed, was 18.5 hours. In seven of the 132 isolates $(5 \%)$, there was non-concordance between the initial Gram stain and subsequent culture. Five of these patients were on adequate empiric treatment although two of these had their treatment altered because of culture results. The remaining two patients started appropriate treatment at the time of the Gram stain result, in the light of clinical findings, likely source, and previous isolates from other sites. The original Gram stains in these cases were reviewed in the light of culture results. In two cases it was felt that a correct identification could have been made on the Gram stain. Details of these nonconcordant isolates are given in (table 2).

Empiric treatment was started at the time the blood culture was taken in 107 (87\%) patients. The average number of antimicrobial agents used for empiric treatment was 1.7 (range 1-4). Treatment was altered on the basis of the Gram stain in $39(36 \%)$ patients and altered again on culture results in 11 patients. Empiric treatment was altered on the basis of culture and sensitivity results alone in 42 $(39 \%)$ of these 107 patients.

Of the $16(13 \%)$ patients who were not treated empirically, treatment was started on the basis of the Gram stain in 13 (five of these were further changed on the basis of culture) and the remaining three patients had treatment started on the basis of culture results alone. There was complete compliance with therapeutic advice given by the microbiology team.

The appropriateness of empiric treatment was assessed in relation to the hospital antibiotic policy. Of 107 patients who received empiric treatment, 58 (54\%) were in accordance with the antibiotic policy and 49 (46\%) were not. These figures were broken down in relation to the underlying source of the bacteraemia (table 3). Where treatment did not com-

Table 2 Isolates with non-concordance between Gram stain and culture

\begin{tabular}{lll}
\hline Initial Gram result & Identification & Source \\
\hline Gram positive diplococci & Acinetobacter sp & Central line \\
Gram positive cocci & Haemophilus aphrophilus & Respiratory \\
Gram negative cocci & Haemophilus influenzae & Respiratory \\
Organisms not seen & Streptococcus pneumoniae & Unknown \\
Organisms not seen & Streptococcus mitis & Soft tissue \\
Organisms not seen & Escherichia coli & Urinary \\
Organisms not seen & Streptococcus bovis & Urinary \\
\hline
\end{tabular}

Table 3 Concordance of empiric treatment with hospital antibiotic policy

\begin{tabular}{lcc}
\hline Underlying source & $\begin{array}{l}\text { Policy } \\
n=58(54 \%)\end{array}$ & $\begin{array}{l}\text { Non-policy } \\
n=49(46 \%)\end{array}$ \\
\hline Urinary tract infection & $26(67 \%)$ & $13(33 \%)$ \\
Central venous cannula & $9(82 \%)$ & $2(18 \%)$ \\
Abdominal/hepatobiliary & $3(27 \%)$ & $8(73 \%)$ \\
Wound/soft tissue & $9(53 \%)$ & $10(47 \%)$ \\
Pneumonia & $5(48 \%)$ & $8(62 \%)$ \\
Meningitis & $1(50 \%)$ & $1(50 \%)$ \\
Unknown & $5(48 \%)$ & $7(62 \%)$ \\
\hline
\end{tabular}

ply with the antibiotic policy, it was found that this was mainly because of the use of excessively broad spectrum agents and in particular to the use of agents with little or no activity against Gram positive organisms. Patients with bacteraemia arising from abdominal and respiratory sources were less likely to receive treatment in accordance with the antibiotic policy.

Of the 49 patients who received empiric treatment not in accordance with the antibiotic policy, $21(43 \%)$ had a blood culture isolate resistant to the agents used. In 18 (86\%) of these this was due to the isolation of a Gram positive organism (Staphylococcus spp 14, Enterococcus spp 3, Bacillus sp 1), where the patient had not received an agent with anti-Gram positive activity. Five of these 49 (10\%) patients had isolates of intermediate susceptibility to the agents used (the empiric agents had some activity against the blood culture isolate, but would not be considered appropriate for effective treatment). Of the 58 patients who received treatment in accordance with the policy, only one $(1.7 \%)$ had a resistant isolate $(p<0.05)$ and none was intermediate.

Of the 107 patients who received empiric treatment, the spectrum of antibiotic treatment was narrowed in $58(54 \%)$. In $20(19 \%)$ of these, treatment was successfully narrowed on the basis of Gram stain morphology alone. The average daily cost of empiric treatment was IR $£ 14.19$, while the cost of directed treatment was IR $£ 8.27$. This represents a $42 \%$ reduction in the daily cost of antibiotic treatment.

Of the $11(9 \%)$ patients who died, 10 had received empiric treatment. In three $(27 \%)$ of these patients death was caused by sepsis alone. In six (55\%), death was due mainly to sepsis but there was a significant underlying illness, and in the remaining two (18\%), death was due to an underlying illness alone. Patients who died were less likely to have received empiric treatment in accordance with the antibiotic policy then those who survived, but this did not reach statistical significance $(p=0.1)$. Seven patients died before Gram stain results were available.

\section{Discussion}

It has been suggested that the availability of broad spectrum antibiotics for empiric treatment should negate much of the clinical impact of a positive blood culture, particularly if it is felt that common blood culture isolates have predictable sensitivities. ${ }^{1}$ This was not the case in our study. Despite the existence of a hospital antibiotic policy that guides empiric treatment, 
$26(24 \%)$ of the 107 patients who received empiric treatment had an isolate that was resistant or had intermediate susceptibilities to the antibiotic(s) used.

The written policy was not adhered to in almost half of the patients studied. Specific infections, services, and classes of antibiotic were identified for future targeted education to improve policy compliance. In common with other hospitals, our policy is based on clinical determination of the likely source of infection to determine appropriate empiric treatment. That this procedure was not followed is demonstrated by the failure to consider the likelihood of Gram positive infection and the consequent excessive use of anti-Gram negative drugs. The failure to predict accurately likely pathogens in bacteriaemias has been demonstrated in a study by Spencely et al in $1979 .^{2}$ In their study, it was found that the expected pathogen could be named by primary care teams in only $15 \%$ of cases. Likewise in a study by Wilkins et al in $1991,{ }^{3}$ the correct microbiological diagnosis was suspected in $93 \%$ of patients, yet $41 \%$ were on suboptimal treatment. Sturm has shown that, even with an agreed policy, microbiological advice on treatment still has a significant impact. ${ }^{4}$ In his study, $1.7 \%$ of patients where microbiology advice was sought, had an isolate resistant to the empiric treatment used compared with $21 \%$ where advice was not sought.

The impact of blood culture results goes beyond the clinical effect on the individual patient. As shown in this study, there was a $42 \%$ reduction in the cost of antibiotic treatment in patients who had received empiric agents. While the daily savings may not appear great, it should be borne in mind that this represents only the individual drug costs. In a study by Doern et al, early reporting of positive blood cultures resulted in a $21.5 \%$ reduction in antibiotic costs. ${ }^{5}$ In the same study the overall cost savings (including laboratory, pharmacy, radiology, accommodation, and other ancillary costs) were estimated at US\$2 403162 over one year at their institution.

Patients on empiric treatment frequently have a large number of agents used over time and in Yeun et al's study, patients had received a mean of 2.7 agents (range 0-11) before microbiological intervention. ${ }^{6}$ In our study, the mean number of empiric agents used before intervention was 1.7 (range 1-4) and it is possible that a larger number of potentially more expensive agents would have been used in the absence of a positive blood culture result. The reduction in drug cost in this study does not include the potential cost of prolonged empiric treatment, which has been shown to far exceed the cost of directed treatment. ${ }^{7}$

By narrowing the spectrum of directed treatment the level of antibiotic resistance within the hospital should be reduced, which in turn leads to further cost reductions. ${ }^{8}$ Because the response to a positive blood culture involves extensive early clinical liaison, it follows that the impact of that result goes beyond that of a mere printed report. Studies have shown that such direct intervention by microbiologist at ward level results in more rational antibiotic use and has a "knock on" educational function in rationalising further empiric antibiotic use. ${ }^{4}$ In addition early intervention is associated with a greater compliance with therapeutic advise. ${ }^{4}$ In a study by Trenholme et al in 1982 there was an overall compliance with therapeutic advice of $96 \%$; however, this compliance dropped to only $80 \%$ when the intervention took place more than 48 hours after the culture became positive. ${ }^{9}$

In conclusion this study demonstrates that positive blood culture results do have an impact on patient treatment. When combined with early clinical liaison, they are associated with more rational treatment, improved outcome, and a reduction in the cost of treatment. This study validates the practice of early reporting of blood culture results and clinical liaison by the microbiology service in the management of bacteraemia.

1 Greenwood D. Antimicrobial susceptibility testing: are we wasting our time? Br f Biomed Sci 1993;50:31-4.

2 Spencely M, Parker MJ, Dewar RAD, Miller DL. The clinical value of microbiological laboratory investigations. $\mathcal{f}$ Infect 1979;1:23-36.

3 Wilkins EGL, Hickey MM, Khoo S, Hale AD, Umasanker $\mathrm{S}$, Thomas $\mathrm{P}$, et al. Northwick Park Infection Consultation Service Part II. Contribution of the service to patient management: an analysis of results between September management: an and July 1990. ₹ Infect 1991;23:47-56.

4 Sturm AW. Rational use of antimicrobial agents and diagnostic microbiology facilities. I Antimicrob Chemother 1988;22:257-60.

5 Doern GV, Vatour R, Gaudet M, Levy B. Clinical impact of in vitro susceptibility testing and bacterial identification. $\mathcal{f}$ Clin Microb 1994;32:1757-62.

6 Yeun KY, Seto WH, Chau PY. An evaluation of inpatient consultations conducted by clinical microbiologists in a teaching hospital. $\mathcal{F}$ Infect 1992;25:29-37.

7 Edwards LD, Levin S, Balagtas R, Lowe P, Landau W, Lepper MH. Ordering patterns and utilization of bacteriologic per MH. Ordering patterns and utilization of bacte

8 Bamberger DM, Dahl SL. Impact of voluntary vs enforced Bamberger DM, Dahl SL. Impact of voluntary vs enforced
compliance of third-generation cephalosporin use in a compliance of third-generation cephalosporin use

9 Trenholme GM, Kaplan RL, Karakusis PH, Stine T, Furher $\mathrm{J}$, Landau $\mathrm{W}$, et al. Clinical impact of rapid identification and susceptibility testing of bacterial blood culture isolates. $\mathcal{f}$ Antimicrob Chemother 1989;27:1342-5. 